\title{
Nonminimal coupling contribution to DIS at low $x$ in Holographic QCD
}

\author{
Artur Amorim, Robert Carcassés Quevedo, and Miguel S. Costa \\ Centro de Física do Porto e Departamento de Física e Astronomia da Faculdade de Ciências da \\ Universidade do Porto, Rua do Campo Alegre 687, 4169-007 Porto, Portugal
}

(Received 22 May 2018; published 12 July 2018)

\begin{abstract}
We consider the effect of including a nonminimal coupling between a $U(1)$ vector gauge field and the graviton Regge trajectory in holographic QCD models. This coupling describes the QCD interaction between the quark bilinear electromagnetic current and the Pomeron. We test this new coupling against DIS data at low Bjorken $x$ and obtain an excellent fit with a chi squared of 1.1 over a very large kinematical range in the photon virtuality $Q^{2}<400 \mathrm{GeV}^{2}$ and for $x<10^{-2}$. The scale of the new dimension full coupling, which arises from integrating higher spin fields, is of order $1-10 \mathrm{GeV}$. This value matches precisely the expectations from effective field theory, which indicate that such corrections are controlled by the mass gap between the spin two and spin four glueballs that are described holographically by the graviton and spin four field in the graviton Regge trajectory, respectively.
\end{abstract}

DOI: 10.1103/PhysRevD.98.026016

\section{INTRODUCTION}

The observation that the Pomeron is dual to the graviton Regge trajectory [1] opened an entirely new approach to the analysis of QCD processes dominated by Pomeron exchange. This fact has been explored in diffractive processes, like low- $x$ deep inelastic scattering (DIS) [2-25], deeply virtual Compton scattering [26], vector meson production [27], double diffractive Higgs production [28], central production of mesons [29] and other inclusive processes [30]. It is now clear that holographic QCD is a valuable tool to model the physics of gluon rich medium, where standard perturbative techniques like the BFKL pomeron [31-33] breakdown.

In this paper we focus on low $x$ DIS, extending the previous work [25]. The basic idea is to construct the holographic Regge theory for the glueball exchange associated with the Pomeron trajectory. In DIS the Pomeron couples to the quark bilinear electromagnetic current $J^{\mu}=\bar{\psi} \gamma^{\mu} \psi$, which is described holographically by the interaction between a bulk $U(1)$ vector gauge field and the graviton Regge trajectory. Here we shall extend the analysis of [25] by allowing for a non-minimal coupling between this gauge field and the higher spin fields in the graviton Regge trajectory. We shall fit the same set of data as in [25], more concretely we fit 249 data points, covering the very large kinematical range of $x<10^{-2}$ and

Published by the American Physical Society under the terms of the Creative Commons Attribution 4.0 International license. Further distribution of this work must maintain attribution to the author(s) and the published article's title, journal citation, and DOI. Funded by SCOAP ${ }^{3}$.
$Q^{2}<400 \mathrm{GeV}^{2}$, where $x$ is the Bjorken $x$ and $Q^{2}$ the photon virtuality. As a result, we manage to improve the quality of our fit from a chi squared per degree of freedom of 1.7 in [25] to an excellent value of 1.1 in the present work.

The existence of such nonminimal coupling between the bulk $U(1)$ gauge field and the graviton Regge trajectory is expected. Starting from the UV high energy limit, the OPE expansion of the two currents, $J_{\mu}(x) J_{\nu}(y)$, contains two OPE coefficients for each spin $J$ symmetric traceless operator associated with the glueballs on the pomeron trajectory, $\mathcal{O}_{J} \sim \operatorname{tr}\left(F_{\mu \alpha_{1}} D_{\alpha_{2}} \ldots D_{\alpha_{J-1}} F_{\alpha_{J}}^{\mu}\right)$. Holographically, and for pure AdS space, this amounts to precisely the same counting when coupling a vector gauge field to the graviton, or to the higher spin fields in the gravity Regge trajectory. Thus we shall consider such non-minimal coupling. In fact, since QCD is not a conformal theory, there is actually more freedom in the choice of such couplings in holographic QCD which, as we shall see, are very much model dependent. For concreteness we shall consider one such coupling, which arises in an effective field theory expansion in the dual QCD string tension. After obtaining the new expression for the DIS structure function $F_{2}\left(x, Q^{2}\right)$ in generic AdS/QCD models, we focus on the specify holographic QCD model of [34-36]. This allows us to put numbers in our expressions that are then tested against available low $x$ DIS data.

\section{HOLOGRAPHIC COMPUTATION OF $\boldsymbol{F}_{2}$ STRUCTURE FUNCTION}

The structure function $F_{2}\left(x, Q^{2}\right)$ is related to the total cross section of the inelastic $\gamma^{*} p \rightarrow X$ process. As discuss in the standard literature (see for instance [37]), defining $\sigma_{T}$ 
and $\sigma_{L}$ to be the cross sections for transverse and longitudinal polarizations, we have

$$
\sigma_{T}+\sigma_{L}=\frac{4 \pi^{2} \alpha}{Q^{2}} F_{2}\left(x, Q^{2}\right)
$$

where $\alpha$ is the fine structure constant. The structure function depends on the photon virtuality $Q^{2}$ and on the Bjorken $x \ll 1$, which we take to be small. Through the optical theorem, this total cross section can be related to the imaginary part of the amplitude $\mathcal{A}$ for elastic forward scattering $\gamma^{*} p \rightarrow \gamma^{*} p$, with the appropriate polarizations. Thus

$$
F_{2}\left(x, Q^{2}\right)=\frac{Q^{2}}{4 \pi^{2} \alpha} \frac{1}{S} \operatorname{Im} \mathcal{A}(s, t=0),
$$

where $s$ and $t$ are the usual Mandelstam variables (in the low $x$ regime, $s=Q^{2} / x$ ). We will compute this amplitude using the AdS/QCD prescription as described below.

First let us define our kinematic variables. We use lightcone coordinates $(+,-, \perp)$, with the flat space metric given by $d s^{2}=-d x^{+} d x^{-}+d x_{\perp}^{2}$, where $x_{\perp} \in \mathbb{R}^{2}$ is a vector in impact parameter space. We take for the large $s$ kinematics of $12 \rightarrow 34$ scattering the following

$$
\begin{aligned}
& k_{1}=\left(\sqrt{s},-\frac{Q^{2}}{\sqrt{s}}, 0\right), \quad k_{3}=-\left(\sqrt{s}, \frac{q_{\perp}^{2}-Q^{2}}{\sqrt{s}}, q_{\perp}\right), \\
& k_{2}=\left(\frac{M^{2}}{\sqrt{s}}, \sqrt{s}, 0\right), \quad k_{4}=-\left(\frac{M^{2}+q_{\perp}^{2}}{\sqrt{s}}, \sqrt{s},-q_{\perp}\right) .
\end{aligned}
$$

where $k_{1}$ and $k_{3}$ are respectively the incoming and outgoing photon momenta. The proton target has mass $M$ and incoming and outgoing momenta $k_{2}$ and $k_{4}$, respectively. For the forward scattering considered in the optical theorem we set $q_{\perp}=0$, so that $k_{1}=-k_{3}$, and we take the same polarization for the incoming and outgoing photon. The possible polarization vectors are

$$
n^{\mu}(\lambda)= \begin{cases}\left(0,0, \epsilon_{\lambda}\right), & \lambda=1,2, \\ (\sqrt{s} / Q, Q / \sqrt{s}, 0), & \lambda=3,\end{cases}
$$

where $\epsilon_{\lambda}$ is just the usual transverse polarization vector.

\section{A. AdS/QCD}

We shall compute the above scattering amplitude using the framework of AdS/QCD. First we present general formulae and then specify to a particular model. As explained in the introduction, we are interested in the Regge limit where the amplitude is dominated by the exchange of the graviton Regge trajectory, which includes fields of even spin $J$. We also need to define our holographic external states. The corresponding Witten diagram is shown in Fig. 1. The upper part of the diagram is related

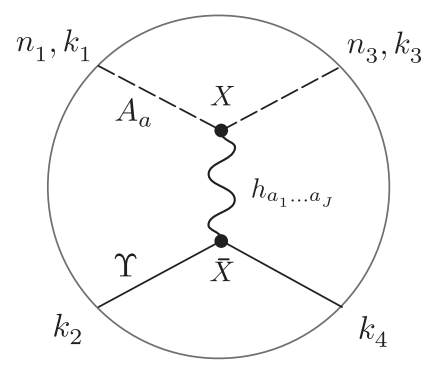

FIG. 1. Tree level Witten diagram representing spin $J$ exchange in a $12 \rightarrow 34$ scattering.

to the incoming and outgoing virtual photons, whereas the bottom part to the proton target.

The holographic dual of QCD will have a dilaton field and a five-dimensional metric, which in the vacuum will have the form

$$
d s^{2}=e^{2 A(z)}\left[d z^{2}+\eta_{\mu \nu} d x^{\mu} d x^{\nu}\right], \quad \Phi=\Phi(z),
$$

for some unknown functions $A(z)$ and $\Phi(z)$. The dilaton is dual to the Lagrangian and the metric to the energymomentum tensor. We shall use greek indices in the boundary, with flat metric $\eta_{\mu \nu}$. We will work with the string frame metric.

In DIS the external photon is a source for the conserved $U(1)$ current $\bar{\psi} \gamma^{\mu} \psi$, where the quark field $\psi$ is associated to the open string sector. The five dimensional dual of this current is a massless $U(1)$ gauge field $A$. We shall assume that this field is made out of open strings and that is nonminimally coupled to the metric, with the following action

$S_{A}=-\frac{1}{4} \int d^{5} X \sqrt{-g} e^{-\Phi}\left(F_{a b} F^{a b}+\beta R_{a b c d} F^{a b} F^{c d}\right)$,

where $F=d A$ and we use the notation $X^{a}=\left(z, x^{\alpha}\right)$ for five-dimensional points. The corresponding equation of motion can be easily derived to be

$$
\nabla_{a}\left[e^{-\Phi}\left(F^{a b}+\beta R^{a b}{ }_{c d} F^{c d}\right)\right]=0 .
$$

The coupling $\beta$ has dimensions of length squared. At this order in derivatives of the fields, we could have other couplings to the Riemann tensor, to derivatives of the dilaton field and also higher derivative terms in the field strength $F$. As we shall see bellow, we will be mostly interested in the coupling to the graviton in the linearised theory, in which case there are only two possible local couplings. Thus, for our purposes the above action is rather general.

We will fix the gauge of the $U(1)$ bulk field to be $D_{a} A^{a}=0$, which gives $A_{z}=0$ and $\partial_{\mu} A^{\mu}=0$. The solution of the equation of motion (7) in this gauge is then

$$
A_{\mu}^{\lambda}(X ; k, \lambda)=n_{\mu}(\lambda) f_{k}(z) e^{i k \cdot x},
$$


where $f_{k}(z)$ solves the differential equation

$$
\left[-Q^{2}+e^{\Phi-A} \partial_{z}\left(e^{A-\Phi} \partial_{z}\right)+\beta \Delta_{\beta}\right] f_{Q}(z)=0,
$$

with

$$
\Delta_{\beta}=-2 e^{-2 A}\left[(-\dot{A} \ddot{A}-\dot{\Phi} \ddot{A}+\ddot{A}) \partial_{z}+\ddot{A} \partial_{z}^{2}-\dot{A}^{2} Q^{2}\right] .
$$

Notice that here, and in the remainder of this paper, we shall denote derivatives with respect to $z$ with a dot. The momentum $k$ and the polarization vector $n(\lambda)$ satisfy

$$
k^{2}=Q^{2}, \quad n_{z}(\lambda)=0, \quad k \cdot n(\lambda)=0,
$$

where the boundary polarization is given by (4). We choose as UV boundary condition $f(0)=1$ which gives the nonnormalizable solution, since the off-shell photon acts as a source for the quark bilinear current $\bar{\psi} \gamma^{\mu} \psi$. Finally, let us note that, for the computation of the Witten diagram in Fig. 1, it is convenient to compute the field strength of a given mode

$$
\begin{aligned}
& F_{\mu \nu}(X ; k, \lambda)=2 i k_{[\mu} n_{\nu]}(\lambda) f_{Q}(z) e^{i k \cdot x}, \\
& F_{z \mu}(X ; k, \lambda)=n_{\mu}(\lambda) \dot{f}_{Q}(z) e^{i k \cdot x},
\end{aligned}
$$

where $Q^{2}=k^{2}$.

For the proton target we consider a scalar field $\Upsilon$ that represents an unpolarized proton described by a normalizable mode of the form

$$
\Upsilon(X ; p)=v_{m}(z) e^{i p \cdot x}
$$

where $p$ is the momentum and $m^{2}=-p^{2}$. As explained in detail in [25], the specific details of the function will not be important because it will appear in an integral that can be absorbed in the coupling between the Pomeron and the proton.

\section{B. Nonminimal coupling}

To compute the Witten diagram of Fig. 1, we need to consider the interaction between the external scattering states and the spin $J$ fields in the graviton Regge trajectory. Thus, the higher spin field comes from the closed string sector while the external fields come from the open sector.

First we consider the coupling between the $U(1)$ gauge field and the graviton. In Einstein-Maxwell theory, and for $A d S$ or flat space, it is well known that there are only two possible cubic couplings between these fields, namely

$$
F^{a c} F^{b}{ }_{c} h_{a b}, \quad F^{a c} F^{b d} \nabla_{c} \nabla_{d} h_{a b},
$$

where $h_{a b}$ is the metric fluctuation. The present case, however, is less restrictive because we have an additional scalar field and also because space-time is not maximally symmetric. To understand this better, let us linearize the action (6) around the background metric, that is, we write $g_{a b}=\bar{g}_{a b}+h_{a b}$. Setting $h=h^{a}{ }_{a}=0$ we have the cubic couplings

$$
\begin{aligned}
\delta S= & -\frac{1}{2} \int d^{5} X \sqrt{-\bar{g}} e^{-\Phi}\left(F^{a b} F_{b}^{c} h_{a c}+\frac{\beta}{2} h_{a p} \bar{R}_{b c d}^{p} F^{a b} F^{c d}\right. \\
& \left.-\beta F^{a c} F^{b d} \bar{\nabla}_{a} \bar{\nabla}_{b} h_{c d}\right)
\end{aligned}
$$

To study the graviton Regge trajectory in the background (5) we need to decompose the metric in $S O(1,3)$ irreducible representations. We will be only interested in the graviton TT components $h_{\alpha \beta}$, satisfying $\partial^{\alpha} h_{\alpha \beta}=0$ and $h_{\alpha}^{\alpha}=0$, and we set $h_{z \alpha}=0=h_{z z}$. Using that $R_{\alpha \mu \beta \nu}=\dot{A}^{2} e^{2 A}\left(\eta_{\alpha \nu} \eta_{\mu \beta}-\right.$ $\left.\eta_{\alpha \beta} \eta_{\mu \nu}\right)$ and $R_{\alpha z \beta z}=-\ddot{A} e^{2 A} \eta_{\alpha \beta}$ in the background (5), and computing the covariant derivatives, we obtain

$$
\begin{aligned}
\delta S= & -\frac{1}{2} \int d^{5} X \sqrt{-\bar{g}} e^{-\Phi}\left[F^{\alpha \mu} F^{\beta}{ }_{\mu}\left(1-\beta e^{-2 A} \dot{A} \partial_{z}\right)\right. \\
& -\beta F^{\alpha \mu} F^{\beta \nu} \partial_{\mu} \partial_{\nu}-2 \beta F^{\alpha z} F^{\beta \nu}\left(\partial_{z}-2 \dot{A}\right) \partial_{\nu} \\
& \left.+F^{\alpha z} F^{\beta}{ }_{z}\left(1-\beta e^{-2 A}\left(\partial_{z}^{2}-3 \dot{A} \partial_{z}+2 \dot{A}^{2}\right)\right)\right] h_{\alpha \beta} .
\end{aligned}
$$

Notice that in the AdS case $(A=-\log z)$ these couplings reduce to the two allowed couplings in (14). However, in the present case there are more possibilities. For example, other contractions with the Riemann tensor will give different functions multiplying the same tensor structures in the couplings. We may also use derivatives of the scalar field to contract with the field strength. For simplicity, the approach we follow in this work will be to focus on the coupling given by the action (6). Our aim is to test whether this type of corrections are important in describing DIS using holographic QCD.

Next we wish to generalize the previous coupling to case of the cubic interaction between the gauge field and a symmetric, transverse and traceless spin $J$ field, $h_{a_{1} \ldots a_{J}}$. The Pomeron trajectory includes such higher spin fields of even $J$. Again there are several possibilities, but we shall focus on the simplest extension of the two couplings to the graviton considered above. The first term is the minimal coupling term, which can be generalized to

$$
\kappa_{J} \int d^{5} X \sqrt{-\bar{g}} e^{-\Phi} F^{a_{1} b} \bar{\nabla}^{a_{2}} \ldots \bar{\nabla}^{a_{J-1}} F^{a_{J}} h_{a_{1} \ldots a_{J}} .
$$

The transverse condition of $h_{a_{1} \ldots a_{J}}$ guarantees that this term is unique up to dilaton derivatives. For the nonminimal coupling we will write 


$$
\begin{gathered}
\beta_{J} \int d^{5} X \sqrt{-\bar{g}} e^{-\Phi}\left(F^{c a_{1}} \bar{\nabla}^{a_{2}} \ldots \bar{\nabla}^{a_{J-1}} F^{a_{J} d} \bar{\nabla}_{c} \bar{\nabla}_{d}\right. \\
\left.+\frac{1}{2} F^{a_{1} b} \bar{\nabla}^{a_{2}} \ldots \bar{\nabla}^{a_{J-1}} F^{c d} R^{a_{J}} b c d\right) h_{a_{1} \ldots a_{J}}
\end{gathered}
$$

We remark that in both expressions (17) and (18) the way we distribute the covariant derivatives acting on the field strength is important. After integrating by parts such a covariant derivative, we are left with an extra term in the derivative of the background dilaton field. However, these terms will have a component of the higher spin field along the $z$ direction, which can be dropped in the case of the Pomeron.

Next we need to decompose the spin $J$ fields in $S O(1,3)$ irreducible representations. In the Regge limit we are only interested in the TT components of these fields, that is in $h_{\alpha_{1} \ldots \alpha_{J}}$ with $\partial^{\nu} h_{\nu \alpha_{2} \ldots \alpha_{J}}=0$ and $h_{\nu \alpha_{3} \ldots \alpha_{J}}^{\nu}=0$. From now on we will assume these two conditions. Thus for the minimal coupling (17) we obtain simply

$$
\begin{aligned}
& \kappa_{J} \int d^{5} X \sqrt{-\bar{g}} e^{-\Phi}\left(F^{\alpha_{1} \mu} \partial^{\alpha_{2}} \ldots \partial^{\alpha_{J-1}} F_{\mu}^{\alpha_{J}}\right. \\
& \left.+F^{\alpha_{1} z} \partial^{\alpha_{2}} \ldots \partial^{\alpha_{J-1}} F^{\alpha_{J}}\right) h_{\alpha_{1} \ldots \alpha_{J}}
\end{aligned}
$$

For the nonminimal coupling (18) we obtain after a cumbersome computation

$$
\begin{aligned}
& \beta_{J} \int d^{5} X \sqrt{-\bar{g}} e^{-\Phi}\left[F^{z \alpha_{1}} \partial^{\alpha_{2}} \cdots \partial^{\alpha_{J-1}} F^{\alpha_{J}}{ }_{z} \mathcal{D}_{\|}^{J}\right. \\
& \quad+F^{\mu \alpha_{1}} \partial^{\alpha_{2}} \cdots \partial^{\alpha_{J-1}} F^{\alpha_{J} \nu}\left(e^{2 A} \mathcal{D}_{\perp}^{J} \eta_{\mu \nu}+\partial_{\mu} \partial_{\nu}\right) \\
& \left.\quad+2 F^{\mu \alpha_{1}} \partial^{\alpha_{2}} \cdots \partial^{\alpha_{J-1}} F^{\alpha_{J} z}\left(\partial_{z}-J \dot{A}\right) \partial_{\mu}\right] h_{\alpha_{1} \cdots \alpha_{J}},
\end{aligned}
$$

where

$$
\begin{aligned}
\mathcal{D}_{\perp}^{J}= & e^{-2 A} \dot{A}\left(\partial_{z}-(J-2) \dot{A}\right), \\
\mathcal{D}_{\|}^{J}= & e^{-2 A}\left(\partial_{z}^{2}-(2 J-1) \dot{A} \partial_{z}\right. \\
& \left.-(J-2) \ddot{A}+J(J-1) \dot{A}^{2}\right) .
\end{aligned}
$$

For $J=2$ this coupling reduces to the graviton nonminimal coupling given in (16).

For the scalar field $\Upsilon$ we will consider a minimal coupling with spin $\mathrm{J}$ closed string fields

$$
\bar{\kappa}_{J} \int d^{5} X \sqrt{-\bar{g}} e^{-\Phi}\left(\Upsilon \nabla^{a_{1}} \ldots \nabla^{a_{J}} \Upsilon\right) h_{a_{1} \ldots a_{J}}
$$

Again, this coupling is unique up to derivatives of the dilaton field that are subleading in the Regge limit. Focusing on the TT part of the spin $J$ field, we are left with the single coupling

$$
\bar{\kappa}_{J} \int d^{5} X \sqrt{-\bar{g}} e^{-\Phi}\left(\Upsilon \partial^{\alpha_{1}} \ldots \partial^{\alpha_{J}} \Upsilon\right) h_{\alpha_{1} \ldots \alpha_{J}}
$$

\section{Witten diagram in Regge limit}

The scattering amplitude will have a contribution from the minimal and the nonminimal coupling. The contribution of the minimal coupling to the structure function $F_{2}$ is presented and described in [25]. Here we shall compute the contribution of the nonminimal coupling (20) to the exchange of a spin $J$ field, corresponding to the Witten diagram in Fig. 1. Using the Regge kinematics (3) and taking as external states $F_{i}^{a b}(X)$ for $i=1,3$ and $\Upsilon_{j}(\bar{X})$ for $j=2$, 4, we obtain for forward scattering the expression

$$
\begin{aligned}
\beta_{J} \bar{\kappa}_{J} & \sum_{\lambda=1}^{3} \int d^{5} X d^{5} \bar{X} \sqrt{-g} \sqrt{-\bar{g}} e^{-\Phi} e^{-\bar{\Phi}} \Upsilon_{2}\left(\bar{\partial}^{-}\right)^{J} \Upsilon_{4} \\
& \times\left[F_{1}^{+z}\left(\partial^{+}\right)^{J-2} F_{3 z}^{+} \mathcal{D}_{\|}^{J}+F_{1}^{+\mu}\left(\partial^{+}\right)^{J-2} F_{3 \mu}^{+} \mathcal{D}_{\perp}^{J}\right] \\
& \times \Pi_{+\ldots+,-\cdots-}(X, \bar{X}),
\end{aligned}
$$

where bars denote quantities evaluated at $\bar{X}$. Notice that the couplings involving derivatives along the boundary in (20) vanish for forward scattering. Using (12) and (13) for the external states and performing the sum over polarisations we find

$$
\begin{gathered}
-\beta_{J} \bar{\kappa}_{J} s^{J} \int d^{5} X d^{5} \bar{X} \sqrt{-g} \sqrt{-\bar{g}} e^{-\Phi-\bar{\Phi}-2(J+1) A-2 J \bar{A}} \\
\times v_{m}^{2}(\bar{z})\left(f_{Q}^{2}(z) \mathcal{D}_{\perp}^{J}+\frac{\dot{f}_{Q}^{2}(z)}{Q^{2}} \mathcal{D}_{\|}^{J}\right) \Pi_{+\cdots+,-\cdots-}
\end{gathered}
$$

We remark that the terms with $\mathcal{D}_{\perp}^{J}$ and with $\mathcal{D}_{\|}^{J}$ are, respectively, the leading contribution arising from the transverse and longitudinal polarizations, therefore justifying our notation.

Performing the change of variable $w=x-\bar{x}$, setting $l_{\perp}=x_{\perp}-\bar{x}_{\perp}, t=-q_{\perp}^{2}$ and defining the transverse propagator at zero momentum transfer by

$$
\begin{array}{r}
\int \frac{d w^{+} d w^{-} d^{2} l_{\perp}}{2} \Pi_{+\cdots+,-\cdots-}(w, z, \bar{z}) \\
=-\frac{i}{2^{J}} e^{(J-1)(A+\bar{A})} G_{J}(z, \bar{z}, t=0),
\end{array}
$$

we finally obtain

$$
\begin{aligned}
& i \frac{\beta_{J} \bar{\kappa}_{J} S^{J}}{2^{J}} V \int d z d \bar{z} e^{-\Phi-\bar{\Phi}-2 J(A+\bar{A})+3 A+5 \bar{A}} v_{m}^{2}(\bar{z}) \\
& \quad \times\left(f_{Q}^{2}(z) \mathcal{D}_{\perp}^{J}+\frac{\dot{f}_{Q}^{2}(z)}{Q^{2}} \mathcal{D}_{\|}^{J}\right)\left[e^{(J-1)(A+\bar{A})} G_{J}(z, \bar{z}, 0)\right] .
\end{aligned}
$$

where the space-time volume $V$ comes from the delta function momentum conservation that we imposed from the beginning on the external states. Now we proceed as in [25] and write a spectral representation for the transverse propagator 


$$
G_{J}(z, \bar{z}, t)=e^{B+\bar{B}} \sum_{n} \frac{\psi_{n}(J, z) \psi_{n}^{*}(J, \bar{z})}{t_{n}(J)-t}
$$

where $\psi_{n}(J, z)$ are the normalizable modes associated to the spin $J$ fields. The function $B(z)$ depends on the particular holographic QCD model. We will fix it later in order to perform fits to data.

\section{Regge theory}

In order to get the total amplitude we need to sum over even spin $J$ fields with $J \geq 2$. Then we can apply a Sommerfeld-Watson transform

$$
\frac{1}{2} \sum_{J \geq 2}\left(s^{J}+(-s)^{J}\right)=-\frac{\pi}{2} \int \frac{d J}{2 \pi i} \frac{s^{J}+(-s)^{J}}{\sin \pi J},
$$

which requires analytic continuation of the amplitude for spin $J$ exchange to the complex J-plane. We assume that the $\mathrm{J}$-plane integral can be deformed from the poles at even $\mathrm{J}$, to the poles $J=j_{n}(t)$ defined by $t_{n}(J)=t$. The scattering domain of negative $t$ contains these poles along the real axis for $J<2$. The scattering amplitude for $t=0$ is then

$$
\begin{aligned}
A(s, 0)= & \sum_{n} h_{n} s^{j_{n}} \int d z e^{-\Phi} e^{A\left(-2 j_{n}+3\right)} \\
& \times\left(f_{Q}^{2} \mathcal{D}_{\perp}^{j_{n}(0)}+\frac{\dot{f}_{Q}^{2}}{Q^{2}} \mathcal{D}_{\|}^{j_{n}(0)}\right)\left[e^{A\left(j_{n}(0)-1\right)} e^{B} \psi_{n}\left(j_{n}(0), z\right)\right],
\end{aligned}
$$

with $h_{n}$ defined as

$$
\begin{aligned}
h_{n}= & -\frac{\pi}{2} \frac{\beta_{j_{n}(0)} \bar{\kappa}_{j_{n}(0)}}{2^{j_{n}(0)}}\left(i+\cot \frac{\pi j_{n}(0)}{2}\right) j_{n}^{\prime}(0) \\
& \times \int d \bar{z} e^{\bar{A}\left(4-j_{n}(0)\right)} e^{-\bar{\Phi}} e^{\bar{B}} v_{m}^{2}(\bar{z}) \psi_{n}^{*}\left(j_{n}(0), \bar{z}\right) .
\end{aligned}
$$

Finally, the action of the differential operators on the functions of $z$ allows us to rewrite the forward scattering amplitude as

$$
\begin{aligned}
A(s, 0)= & \sum_{n} h_{n} s^{j_{n}} \int d z e^{-(j-2) A+B-\Phi} \\
& \times\left(f_{Q}^{2} \tilde{\mathcal{D}}_{\perp}^{j_{n}(0)}+\frac{\dot{f}_{Q}^{2}}{Q^{2}} \tilde{\mathcal{D}}_{\|}^{j_{n}(0)}\right) \psi_{n}\left(j_{n}(0), z\right),
\end{aligned}
$$

with

$$
\begin{aligned}
\tilde{\mathcal{D}}_{\perp} & =e^{-2 A}\left(\dot{A} \partial_{z}+\dot{A}^{2}+\dot{A} \dot{B}\right), \\
\tilde{\mathcal{D}}_{\|} & =e^{-2 A}\left(\partial_{z}^{2}-(\dot{A}-2 \dot{B}) \partial_{z}+\ddot{B}+\ddot{A}+\dot{B}^{2}-\dot{A} \dot{B}\right) .
\end{aligned}
$$

\section{E. $\boldsymbol{F}_{2}$ structure function}

The DIS structure function can be written in Regge theory in the following form

$$
F_{2}\left(x, Q^{2}\right)=\sum_{n}\left(f_{n}^{\mathrm{MC}}\left(Q^{2}\right)+f_{n}^{\mathrm{NMC}}\left(Q^{2}\right)\right) x^{1-j_{n}}
$$

where we separated the contributions from the minimal and nonminimal couplings between the graviton trajectory and the $U(1)$ current that arise from the holographic computation. In [25], for $B=\Phi-A / 2$, we showed that

$f_{n}^{\mathrm{MC}}\left(Q^{2}\right)=g_{n} Q^{2 j_{n}} \int d z e^{-\left(j_{n}-\frac{3}{2}\right) A}\left(f_{Q}^{2}+\frac{\dot{f}_{Q}^{2}}{Q^{2}}\right) \psi_{n}$.

Using the definitions (1) and (2), we may take the imaginary part of the forward scattering (33), to obtain the contribution from the nonminimal coupling

$f_{n}^{\mathrm{NMC}}\left(Q^{2}\right)=\tilde{g}_{n} Q^{2 j_{n}} \int d z e^{-\left(j_{n}-\frac{3}{2}\right) A}\left(f_{Q}^{2} \tilde{\mathcal{D}}_{\perp}+\frac{\dot{f}_{Q}^{2}}{Q^{2}} \tilde{\mathcal{D}}_{\|}\right) \psi_{n}$,

where $\tilde{g}_{n}=\operatorname{Im}\left(h_{n}\right) /\left(4 \pi^{2} \alpha\right)$. Both constants $g_{n}$ and $\tilde{g}_{n}$ are used as fitting parameters in our setup, thus the details of holographic wave function for the proton are not important in the fit. Notice that the $g_{n}$ and $\tilde{g}_{n}$ do not have the same dimensions, indeed comparing both complings we see that $\left[\tilde{g}_{n} / g_{n}\right]=L^{2}$. Formula (37) is one of the main results of this paper.

\section{F. Improved holographic QCD}

To test the above ideas against experimental data we need to consider a concrete QCD holographic model. As in our previous work [25], we shall consider the improved holographic QCD model introduced in [34-36]. This fixes the background fields $A(z)$ and $\Phi(z)$, which give an approximate dual description of the $\mathrm{QCD}$ vacuum.

Next we need to consider the equation of motion for the spin $J$ fields that are dual to the twist two operators, whose exchange gives the dominate contribution in DIS at low $x$. This equation is then analytically continue in $J$, in order to do the Sommerfeld-Watson transform in Regge theory. This procedure was described in detail in [25], so we will not repeat it here. The upshot is that the function $B$ introduced in (28) to define the transverse propagator is given by $B=\Phi-A / 2$ and the normalizable modes of the spin $J$ field $\psi_{n}(z)$ solve a Schrödinger problem

$$
\left(-\frac{d^{2}}{d z^{2}}+U_{J}(z)\right) \psi_{n}(z)=t_{n} \psi_{n}(z)
$$

where 


$$
\begin{aligned}
U_{J}(z)= & \frac{3}{2}\left(\ddot{A}-\frac{2}{3} \ddot{\Phi}\right)+\frac{9}{4}\left(\dot{A}-\frac{2}{3} \dot{\Phi}\right)^{2} \\
& +(J-2) e^{-2 A}\left[\frac{2}{l_{s}^{2}}\left(1+\frac{d}{\sqrt{\lambda}}\right)+\frac{J+2}{\lambda^{4 / 3}}\right. \\
& \left.+e^{2 A}\left(a \ddot{\Phi}+b\left(\ddot{A}-\dot{A}^{2}\right)+c \dot{\Phi}^{2}\right)\right],
\end{aligned}
$$

where the first line represents the potential for the graviton and the remaining proposed terms deform the graviton potential. This potential is analytically continued in $J$ in such a way that the value of the intercept $J=j_{n}$ is obtained when the $n$th eigenvalue satisfies $t_{n}(J)=0$.

The constants $l_{s}, a, b, c$, and $d$ are used as fitting parameters and will be adjusted such that the best match with $F_{2}\left(x, Q^{2}\right)$ data is achieved. In particular, from the low energy effective string theory perspective, $l_{s}$ is related to the string tension; $d$ is related to the anomalous dimension curve of the twist 2 operators, or it can also be thought as encoding the information of how the masses of the closed strings excitations are corrected in a slightly curved background; the constants $a, b$, and $c$ encode the first order derivative expansion of a presumed string field theory lagrangian; $\lambda=e^{\Phi}$ is the 't Hooft coupling.

\section{DATA ANALYSIS}

With the previously described setup we proceed to find the best values for the potential parameters $l_{s}, a, b, c$, and $d$, as well as for the coupling values $\beta, g_{n}$ and $\tilde{g}_{n}$ that better fit the data. We look, as usual, for the best set of parameter values such that the sum of the weighted difference squared between experimental data and model predicted values is minimum, using as weight the inverse of the experimental uncertainty. Since this is a highly nontrivial numerical optimization problem in which we do not known explicitly the gradient of the function to be optimized, we use the Nelder-Mead algorithm, using $R$ language, and try with different starting points in the parameter space. We have found that the inclusion of the nonminimal coupling contribution considerable decreases the convergence ratio of the minimizing routine compared with the case where only the minimal coupling case is used, consistent with the fact that the new function to optimize has a much rougher landscape. Our best fit results for $F_{2}\left(x, Q^{2}\right)$ are presented in Fig. 2. In this fit we considered values of $x$ in the range $x<10^{-2}$, and of the photon virtuality $Q^{2}<400 \mathrm{GeV}^{2}$. This gives a total number of 249 data points. The $\chi_{\text {d.o.f }}^{2}$ for this fit is 1.13. As in our previous work, aiming to make a consistent model for the soft Pomeron, we have forced the intercept of the second trajectory to be around $j_{1}=1.09$. This is achieved penalizing those set of parameters which give a different second intercept by adding a term of the type $10^{4}\left(j_{1}-1.09\right)^{2}$ to the function to be optimized. The correspondent Regge trajectories can be seen in Fig. 3 .

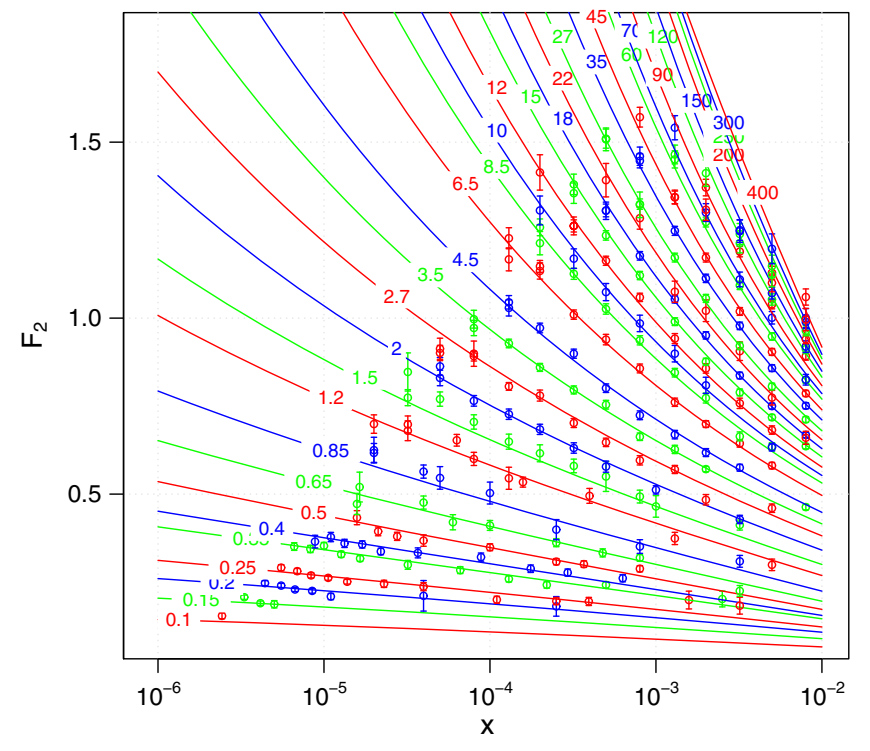

FIG. 2. Structure function $F_{2}\left(Q^{2}, x\right)$. Experimental points vs prediction of this work with a $\chi_{\text {d.o.f }}^{2}=1.1$. Each line corresponds to a given $Q^{2}\left(\mathrm{GeV}^{2}\right)$ as indicated.

The values of the parameters that give the best fit are summarized in Table I. We would like to understand the scale defined by the nonminimal coupling. The best fit fixes the value of this coupling in the equation of motion (7) for the $U(1)$ gauge field to be $\beta=0.026 \mathrm{GeV}^{-2}$. This numerical value correspond to an energy of $6 \mathrm{GeV}$ and therefore the energy scale associated with this correction is in the range of 1-10 GeV. We may also look at the ratio between the constants $g_{n}$ and $\tilde{g}_{n}$, given by,

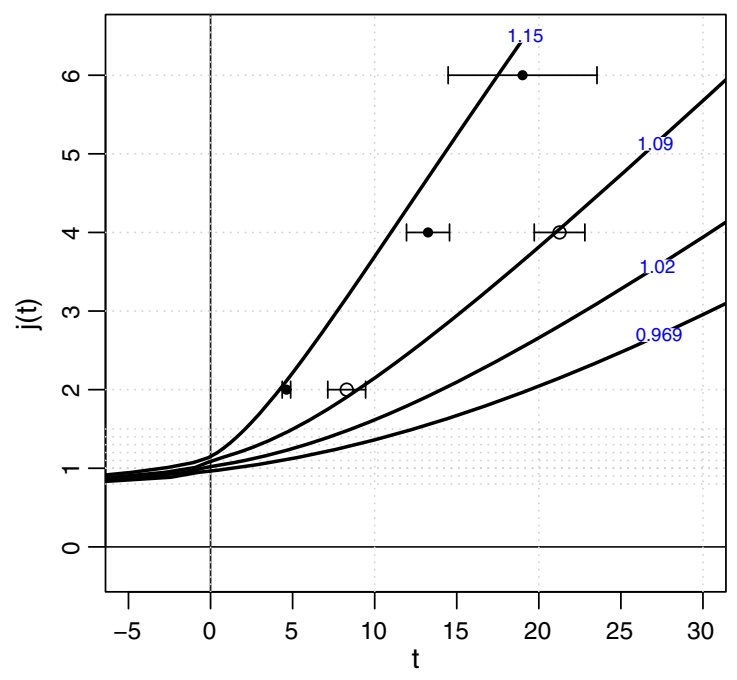

FIG. 3. Regge trajectories compared with glueball masses from lattice simulations $[38,39]$. Shown are also the values we obtained for the intercept of each trajectory. Configurations that give the soft Pomeron intercept $j_{1}=1.09$ were favored in the fitting process. 
TABLE I. Values of the parameters for the best fit found. All parameters are dimensionless except for $\left[l_{s}\right]=L,[\beta]=L^{2}$ and $\left[\tilde{g}_{i}\right]=L^{2}$. Numerical values are expressed in $\mathrm{GeV}$ units.

\begin{tabular}{lccccc}
\hline \hline Parameter & Value & Couplings & Value & Couplings & Value $\times 10$ \\
\hline$l_{s}^{-1}$ & 6.93 & $g_{0}$ & -0.154 & $\tilde{g}_{0}$ & 0.707 \\
$\mathrm{a}$ & -4.68 & $g_{1}$ & -0.424 & $\tilde{g}_{1}$ & -0.378 \\
$\mathrm{~b}$ & 4.85 & $g_{2}$ & 2.12 & $\tilde{g}_{2}$ & -2.48 \\
$\mathrm{c}$ & 0.665 & $g_{3}$ & -0.721 & $\tilde{g}_{3}$ & 3.63 \\
$\mathrm{~d}$ & -0.328 & & & & \\
$\beta$ & -0.026 & & & & \\
\hline \hline
\end{tabular}

$$
\frac{\tilde{g}_{n}}{g_{n}}=\frac{\beta_{j_{n}(0)}}{\kappa_{j_{n}(0)}}
$$

which has dimensions length ${ }^{2}$. This follows from taking the imaginary part of (32) and from the fact that $g_{n}$ has a similar expression [25]. Looking at Table I it is simple to see that the ratio $\tilde{g}_{n} / g_{n}$, for each $n$, is also in the energy range of 1-10 GeV. The scale of the nonminimal coupling should be associated with the mass gap between the spin 2 and spin 4 glueballs, that arise from the spectrum of the bulk graviton and spin 4 field, respectively. Indeed this is precisely the size of the gap observed in the glueball spectrum in Fig. 3.

\section{CONCLUSION}

In this work we considered the contribution of a nonminimal coupling between the $U(1)$ gauge field and the higher spin fields in the graviton Regge trajectory to the holographic computation of the DIS structure function $F_{2}\left(x, Q^{2}\right)$. These non-minimal couplings are expected to be present and to play an important role in theories with higher spin fields. Such terms are controlled by the gap between the graviton and the next higher spin field [40]. Our results are consistent with this expectation since the scale we obtained for the nonminimal coupling has the correct order of magnitude that reproduces the mass difference between the spin 2 and spin 4 glueballs.

With the inclusion of the new coupling the quality of our fit to low $x$ DIS data has improved considerably. In the previous work [25], that considered only the minimal coupling, a $\chi_{\text {d.o.f }}^{2}$ of 1.7 was obtained. With the new coupling we improved this result to a $\chi_{\text {d.o.f }}^{2}$ of 1.1 . We believe this is an important improvement that validates the holographic approach to low $x$ physics. We are reproducing data over a very large kinematical range in the two variables $x$ and $Q^{2}$, fitting a total of 249 points.

One can draw some intuition on how the inclusion of the nonminimal coupling improves the fit to physical data by looking at the Reggeon wave functions. These functions are shown for the hard and soft Pomerons, for the corresponding values of the intercept, in Figs. 4 and 5, respectively. These waves functions are the ground state and first excited

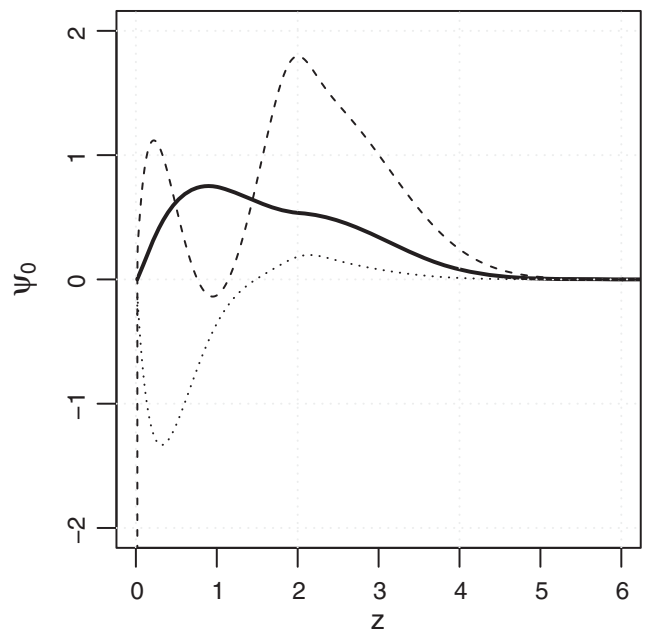

FIG. 4. Hard Pomeron wave function for the best fit found and for its intercept value $J=j_{0}$. The dotted and dashed line represent the action of the operator $\tilde{\mathcal{D}}_{\perp}$ and $\tilde{\mathcal{D}}_{\|}$on the hard Pomeron wave function $\psi_{0}(z)$ respectively. In this plot all the functions have been scaled by a factor of 10 .

state of the associated Schrödinger problem. For the minimal coupling they control the dependence of the structure function in the photon virtuality $Q^{2}$ as can be seen from (36). For the nonminimal coupling they also control the $Q^{2}$ dependence but now the action of the differential operators $\tilde{\mathcal{D}}_{\perp}$ and $\tilde{\mathcal{D}}_{\|}$in (37) changes such dependence to a more oscillating behavior, as can be seen from Figs. 4 and 5. What is not a priori trivial is that this freedom can be used to better fit the data, yielding for the scale of nonminimal coupling precisely the expected order of magnitude (due to the oscillations it could be that this order of magnitude was much smaller, which would seem to contradict the expected value of the gap for higher spin glueballs).

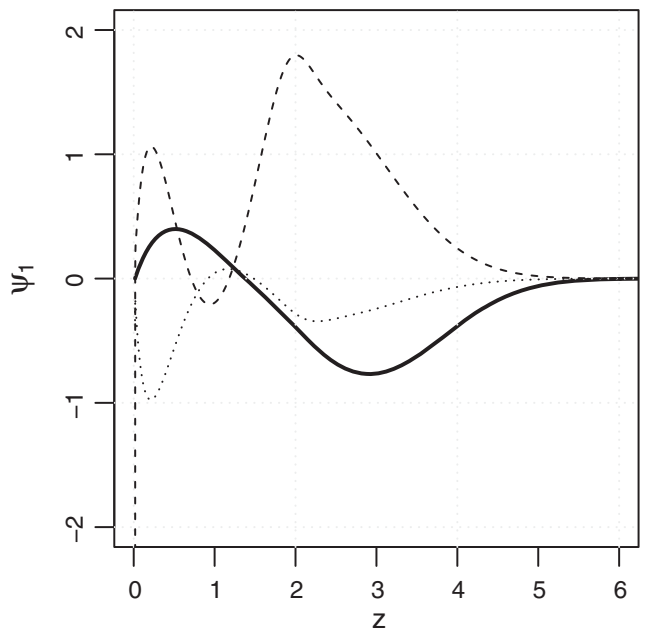

FIG. 5. Same as Fig. 4 but for the soft Pomeron and for its intercept value $J=j_{1}$. 
It seems we are getting closer to a very satisfactory holographic description of low $x$ data. There are two immediate questions that we believe deserve some further attention. As a working example we have been considering the improved holographic QCD model of [34-36]. We take this model as our QCD vacuum, and then introduce higher spins fields for which we do Regge theory. Clearly we should study to which extent other models can also be used to reproduce the data here analysed. Our expectation is that holography is very appropriate to study processes dominated by Pomeron exchange, so that other models that are close enough to QCD should give similar results. Another interesting point is to extend this analysis to other processes than DIS. Previous studies of deeply virtual Compton scattering (DVSC) and vector meson production could now be revisited, including the nonminimal coupling here considered, to attain better fits. For example, in the case of DVSC the cross section depends on three kinematical quantities, namely $x, Q^{2}$ and momentum transfer. Extending the contribution of the nonminimal coupling terms to nonvanishing $t$ gives a very nontrivial dependence that deserves to be looked at.

\section{ACKNOWLEDGMENTS}

We would like to thank A. Ballon Bayona for useful discussions. This research received funding from the grant no. CERN/FIS-PAR/0019/2017 and the Simons Foundation Grant No. 488637 (Simons collaboration on the Nonperturbative bootstrap). A. A. is funded by the International Doctorate Network in Particle Physics, Astrophysics and Cosmology (IDPASC) doctorate programme with the fellowship PD/BD/114158/2016.
[1] R. C. Brower, J. Polchinski, M. J. Strassler, and C.-I. Tan, J. High Energy Phys. 12 (2007) 005.

[2] C. A. Ballon Bayona, H. Boschi-Filho, and N. R. F. Braga, J. High Energy Phys. 03 (2008) 064.

[3] Y. Hatta, E. Iancu, and A. H. Mueller, J. High Energy Phys. 01 (2008) 026.

[4] L. Cornalba and M. S. Costa, Phys. Rev. D 78, 096010 (2008).

[5] B. Pire, C. Roiesnel, L. Szymanowski, and S. Wallon, Phys. Lett. B 670, 84 (2008).

[6] J. L. Albacete, Y. V. Kovchegov, and A. Taliotis, J. High Energy Phys. 07 (2008) 074.

[7] Y. Hatta, J. High Energy Phys. 11 (2008) 057.

[8] E. Levin, J. Miller, B. Z. Kopeliovich, and I. Schmidt, J. High Energy Phys. 02 (2009) 048.

[9] R. C. Brower, M. Djuric, and C.-I. Tan, arXiv:0812.1299.

[10] R. Brower, M. Djuric, and C.-I. Tan, arXiv:0911.3463.

[11] J.-H. Gao and B.-W. Xiao, Phys. Rev. D 80, 015025 (2009).

[12] Y. Hatta, T. Ueda, and B.-W. Xiao, J. High Energy Phys. 08 (2009) 007.

[13] Y. V. Kovchegov, Z. Lu, and A. H. Rezaeian, Phys. Rev. D 80, 074023 (2009).

[14] E. Avsar, E. Iancu, L. McLerran, and D. N. Triantafyllopoulos, J. High Energy Phys. 11 (2009) 105.

[15] L. Cornalba, M. S. Costa, and J. Penedones, J. High Energy Phys. 03 (2010) 1.

[16] F. Dominguez, J. High Energy Phys. 09 (2010) 7.

[17] L. Cornalba, M. S. Costa, and J. Penedones, Phys. Rev. Lett. 105, 072003 (2010).

[18] M. A. Betemps, V.P. Gonçalves, and J. T. de Santana Amaral, Phys. Rev. D 81, 094012 (2010).

[19] J.-H. Gao and Z.-G. Mou, Phys. Rev. D 81, 096006 (2010).

[20] Y. V. Kovchegov, Phys. Rev. D 82, 054011 (2010).

[21] E. Levin and I. Potashnikova, J. High Energy Phys. 08 (2010) 112.
[22] S. K. Domokos, J. A. Harvey, and N. Mann, Phys. Rev. D 80, 126015 (2009).

[23] S. K. Domokos, J. A. Harvey, and N. Mann, Phys. Rev. D 82, 106007 (2010).

[24] R. C. Brower, M. Djuric, I. Sarcevic, and C.-I. Tan, J. High Energy Phys. 11 (2010) 051.

[25] A. Ballon-Bayona, R. Carcassés Quevedo, and M. S. Costa, J. High Energy Phys. 08 (2017) 085.

[26] M. S. Costa and M. Djurić, Phys. Rev. D 86, 016009 (2012).

[27] M. S. Costa, M. Djurić, and N. Evans, J. High Energy Phys. 09 (2013) 1.

[28] R. C. Brower, M. Djuric, and C.-I. Tan, J. High Energy Phys. 09 (2012) 097.

[29] N. Anderson, S. K. Domokos, J. A. Harvey, and N. Mann, Phys. Rev. D 90, 086010 (2014).

[30] R. Nally, T. G. Raben, and C.-I. Tan, J. High Energy Phys. 11 (2017) 075.

[31] V. S. Fadin, E. A. Kuraev, and L. N. Lipatov, Phys. Lett. 60B, 50 (1975).

[32] E. A. Kuraev, L. N. Lipatov, and V. S. Fadin, Zh. Eksp. Teor. Fiz. 72, 377 (1977); [Sov. Phys. JETP 45, 199 (1977)].

[33] I. I. Balitsky and L. N. Lipatov, Yad. Fiz. 28, 1597 (1978); [Sov. J. Nucl. Phys. 28, 822 (1978)].

[34] U. Gürsoy and E. Kiritsis, J. High Energy Phys. 02 (2008) 032.

[35] U. Gürsoy, E. Kiritsis, and F. Nitti, J. High Energy Phys. 02 (2008) 019.

[36] U. Gürsoy, E. Kiritsis, L. Mazzanti, G. Michalogiorgakis, and F. Nitti, Lect. Notes Phys. 828, 79 (2011).

[37] A. C.-S. Robin Devenish, Deep Inelastic Scattering (Oxford University Press, New York, 2004).

[38] H. B. Meyer, arXiv:hep-lat/0508002.

[39] H. B. Meyer and M. J. Teper, Phys. Lett. B 605, 344 (2005).

[40] X. O. Camanho, J. D. Edelstein, J. Maldacena, and A. Zhiboedov, J. High Energy Phys. 02 (2016) 20. 